University of Wollongong

Research Online

Faculty of Engineering and Information

Faculty of Engineering and Information

Sciences - Papers: Part A

Sciences

$1-1-2014$

\title{
Non-parametric radially symmetric mean curvature flow with a free boundary
}

V.-M Wheeler

University of Wollongong, vwheeler@uow.edu.au

Follow this and additional works at: https://ro.uow.edu.au/eispapers

Part of the Engineering Commons, and the Science and Technology Studies Commons

Research Online is the open access institutional repository for the University of Wollongong. For further information contact the UOW Library: research-pubs@uow.edu.au 


\title{
Non-parametric radially symmetric mean curvature flow with a free boundary
}

\author{
Abstract \\ We study the mean curvature flow of radially symmetric graphs with prescribed contact angle on a fixed, \\ smooth hypersurface in Euclidean space. In this paper we treat two distinct problems. The first problem \\ has a free Neumann boundary only, while the second has two disjoint boundaries, a free Neumann \\ boundary and a fixed Dirichlet height. We separate the two problems and prove that under certain initial \\ conditions we have either long time existence followed by convergence to a minimal surface, or finite \\ maximal time of existence at the end of which the graphs develop a curvature singularity. We also give a \\ rate of convergence for the singularity. 2013 Springer-Verlag Berlin Heidelberg.

\section{Keywords} \\ radially, symmetric, non, mean, parametric, curvature, flow, free, boundary \\ Disciplines \\ Engineering | Science and Technology Studies

\section{Publication Details} \\ Wheeler, V. (2014). Non-parametric radially symmetric mean curvature flow with a free boundary. \\ Mathematische Zeitschrift, 276 (1-2), 281-298.
}


Noname manuscript No.

(will be inserted by the editor)

\title{
Non-parametric radially symmetric mean curvature flow with a free boundary
}

\author{
Valentina-Mira Wheeler
}

the date of receipt and acceptance should be inserted later

\begin{abstract}
We study the mean curvature flow of radially symmetric graphs with prescribed contact angle on a fixed, smooth hypersurface in Euclidean space. In this paper we treat two distinct problems. The first problem has a free Neumann boundary only, while the second has two disjoint boundaries, a free Neumann boundary and a fixed Dirichlet height. We separate the two problems and prove that under certain initial conditions we have either long time existence followed by convergence to a minimal surface, or finite maximal time of existence at the end of which the graphs develop a curvature singularity. We also give a rate of convergence for the singularity.
\end{abstract}

Keywords mean curvature flow · free boundary conditions · geometric analysis

Mathematics Subject Classification (2000) 53C44 58 J35

\section{Introduction}

There has been much work on the mean curvature flow problem for immersions and graphs with or without boundary conditions. The study of entire graphs by Ecker and Huisken [6,7] provides a detailed exposition including a long time existence theorem for Lipschitz initial data. The non-parametric mean curvature flow of graphs with either a ninety degree contact angle or Dirichlet boundary condition on cylindrical domains has been studied by Huisken [10] and provides a long time existence and convergence to minimal surfaces theorem. Also we want to mention the work of Altschuler and $\mathrm{Wu}$ [1] which allows

Valentina-Mira Wheeler

Fachbereich Mathematik und Informatik, Freie Universität, Arnimallee 3, 14195 Berlin, Germany,

Current: Institute for Mathematics and its Applications, University of Wollongong, Northfields Ave, Wollongong, NSW 2522, Australia email: vwheeler@uow.edu.au 
arbitrary contact angle at the fixed boundary for two dimensional graphs and the generalisation to arbitrary intrinsic dimension by Guan [8]. A natural next step in this line of research is to study the mean curvature flow of graphs in time dependent domains with either Neumann or Dirichlet boundary conditions.

This began with a series of works on the mean curvature flow of immersions with free boundary, where a restriction on the angle of contact with a fixed hypersurface in Euclidean space is imposed. The works of Stahl [13] and Buckland [3] provide in special cases finite time blow up of curvature with the rescaled solution asymptotic to a hemisphere, and a monotonicity formula respectively. Also here see the work of Koeller [11].

We study two distinct problems in this paper. The first is the mean curvature flow of radially symmetric graphs with a ninety degree contact angle on a fixed hypersurface in Euclidean space. This is a Neumann boundary value problem on a disc with a time dependent radius in $\mathbb{R}^{n}$. The second, also mean curvature flow of radially symmetric graphs, is defined on a time dependent annulus in $\mathbb{R}^{n}$ with a time dependent Neumann boundary and a fixed Dirichlet height.

Let $x=\left(x_{1}, \ldots, x_{n}\right)$ be a point in $\mathbb{R}^{n}$, with $n \geq 2$ and denote by $y=|x|$ the standard length of the position vector corresponding to $x$. We denote by $\Sigma$ an $n$-dimensional smooth hypersurface without boundary, smoothly embedded in $\mathbb{R}^{n+1}$. The Neumann boundary of the immersions generated by our graphs is included in $\Sigma$.

We only consider hypersurfaces $\Sigma$ which are the union of two rotationally symmetric graphs, $\omega_{\Sigma}^{+}, \omega_{\Sigma}^{-}$where

$$
\begin{aligned}
& \operatorname{Dom}\left(\omega_{\Sigma}^{+}\right)=\operatorname{Dom}\left(\omega_{\Sigma}^{-}\right), \\
& \omega_{\Sigma}^{+}(y) \geq 0, \omega_{\Sigma}^{-}(y) \leq 0, \\
& \omega_{\Sigma}^{+}(y)=0 \Leftrightarrow \omega_{\Sigma}^{-}(y)=0,
\end{aligned}
$$

for all $y$. Each point $X \in \Sigma$ can be written as $X=\left(x, \omega_{\Sigma}(y)\right)$, where $\omega_{\Sigma}$ is either $\omega_{\Sigma}^{+}$or $\omega_{\Sigma}^{-}$. We also impose that the graphs meet vertically, that is

$$
\left\langle\nu_{\Sigma}(y), e_{n+1}\right\rangle=0 \text { when } \omega_{\Sigma}=0
$$

where we denote by $\nu_{\Sigma}$ the normal to $\omega_{\Sigma}$ and $\langle\cdot, \cdot\rangle$ is the standard inner product in $\mathbb{R}^{n+1}$. A convention which we use in the following is that the normal to the fixed hypersurface $\Sigma$ is pointing away from the interior of the moving graphs.

We first consider a free Neumann boundary problem defined on an interval $D(t)=(0, r(t)) \subset \mathbb{R}$ with the Neumann boundary given by the freely moving point $\partial D_{N}(t)=r(t)$. The zero boundary point comes from the fact that our general graph which is generated by the radially symmetric graph is defined on a compact region of $\mathbb{R}^{n}$ with no holes. The origin is treated as a boundary point for the radially symmetric problem but causes no issue whatsoever in the later arguments since by symmetry and smoothness we have that at this point the radially symmetric graph is horizontal. In this case the graphs are moving 
'inside' the hypersurface $\Sigma$. The mean curvature flow of a radially symmetric graph $\omega:(0, r(t)) \times[0, T) \rightarrow \mathbb{R}$ attached to $\Sigma$ is then

$$
\begin{aligned}
& \frac{\partial \omega}{\partial t}=\frac{d^{2} \omega}{d y^{2}} \frac{1}{1+\left(\frac{d \omega}{d y}\right)^{2}}+\frac{d \omega}{d y} \frac{n-1}{y} \quad \text { on } \quad(0, r(t)) \times[0, T), \\
& \left\langle\nu_{\omega}, \nu_{\Sigma}\right\rangle=0 \text { and } \omega(r(t), t)=\omega_{\Sigma}(r(t)) \quad \text { on } \quad r(t) \times[0, T) \text {, } \\
& \exists \lim _{y \rightarrow 0} \frac{1}{y} \frac{d \omega}{d y}(y) \\
& \omega(y, 0)=\omega_{0} \\
& \text { on }(0, r(0)) \text {. }
\end{aligned}
$$

Examples of this include graphs evolving inside a catenoid neck or inside the hole of a torus.

The second case we consider is when besides the Neumann boundary condition we also have a fixed Dirichlet boundary condition. In this setting the domain of the general graph is an annulus and the domain of the radially symmetric graph is an interval away from the origin of the form $D(t)=(r(t), R)$. We denote by $\partial D_{N}(t)=r(t)$ and $\partial D_{D}=R$ the time dependent Neumann boundary and fixed Dirichlet boundary respectively. In this case the graphs are moving 'outside' the fixed hypersurface $\Sigma$. The mean curvature flow of a radially symmetric graph $\omega:(r(t), R) \times[0, T) \rightarrow \mathbb{R}$ intersecting orthogonally a fixed hypersurface $\Sigma$ and attached to a fixed circle is then

$$
\begin{aligned}
& \frac{\partial \omega}{\partial t}=\frac{d^{2} \omega}{d y^{2}} \frac{1}{1+\left(\frac{d \omega}{d y}\right)^{2}}+\frac{d \omega}{d y} \frac{n-1}{y} \quad \text { on } \quad(r(t), R) \times[0, T), \\
& \left\langle\nu_{\omega}, \nu_{\Sigma}\right\rangle=0 \text { and } \omega(r(t), t)=\omega_{\Sigma}(r(t)) \quad \text { on } \quad r(t) \times[0, T) \text {, } \\
& \omega(R, t)=z_{0} \quad \text { on }[0, T), \\
& \omega(y, 0)=\omega_{0} \quad \text { on }(r(0), R) .
\end{aligned}
$$

Examples of this include graphs evolving outside a sphere, ellipsoid, and so on.

In this paper we present three main results, giving sufficient conditions for long time existence and convergence to minimal surfaces, and the development of a finite time singularity.

The two theorems of long time existence separate our problems into two major cases. Both of them rely on uniform bounds on the height and the gradient of the radially symmetric graphs. The first theorem provides sufficient conditions for the height to be bounded by the initial values, in contrast with the second theorem, which bounds the height by a (possibly very large) constant. In the first theorem we also separate two cases, depending on the type of problem and also on the conditions imposed on the surface of contact $\Sigma$.

Theorem 1.1 (Long time existence with height bounded by initial values). Let $\Sigma$ and the graph function $\omega_{0}$ be defined as above. Assume that there exists a constant $C$ such that $\sup \left|\omega_{0}\right| \leq C$ and define $\operatorname{Dom}(C)=\left\{y \in \operatorname{Dom}\left(\omega_{\Sigma}\right)\right.$ : $\left.\left|\omega_{\Sigma}(y)\right| \leq C\right\}$. Then: 
(a) if

$$
\begin{aligned}
\omega_{\Sigma}(y) \frac{d \omega_{\Sigma}}{d y}(y) & \geq 0 & & \text { for all } y \in \operatorname{Dom}(C), \text { and } \\
\frac{d \omega_{\Sigma}}{d y}(y) & \neq 0 & & \text { for all } y \in \operatorname{Dom}(C),
\end{aligned}
$$

and if $\operatorname{Dom}(C)$ is bounded then there exists a solution to the problem (1) for all times with $|\omega(y, t)| \leq C$ and it converges to a minimal surface as $t \rightarrow \infty$;

(b) if the domain of the initial graph is $D(0)=(r(0), R)$, where $R \notin$ $\operatorname{Dom}\left(\omega_{\Sigma}\right)$ and if there exists a positive constant $r_{\Sigma}=\sup \operatorname{Dom}\left(\omega_{\Sigma}\right) \in[r(0), R)$ such that $\omega_{\Sigma}\left(r_{\Sigma}\right)=0$ and

$$
\begin{aligned}
\omega_{\Sigma}(y) \frac{d \omega_{\Sigma}}{d y}(y) & \leq 0 & & \text { for all } y \in \operatorname{Dom}(C), \\
\frac{d \omega_{\Sigma}}{d y}(y) & \neq 0 & & \text { for all } y \in \operatorname{Dom}(C),
\end{aligned}
$$

and finally also assuming that the initial graph satisfies the compatibility condition $\left.H\left(\omega_{0}\right)\right|_{y=R}=0$, there exists a solution to the problem (2) for all times with $|\omega(y, t)| \leq C$ and it converges to a minimal surface as $t \rightarrow \infty$.

Our next result gives long time existence for solutions of (2), but with a different set of initial conditions. These conditions do not imply that the height remains bounded by initial values.

Theorem 1.2 (Long time existence without an optimal height bound). Let $\Sigma$ and the graph function $\omega_{0}:(r(0), R) \rightarrow \mathbb{R}$ be as above. Assume that there exists a global constant $C$ such that $\sup \left|\omega_{0}\right| \leq C$ and $\omega_{\Sigma}$ is taken such that $R \in \operatorname{Dom}\left(\omega_{\Sigma}\right)$ and $\left|\omega_{\Sigma}(R)\right|>C$. Assume also that there exists a positive constant $r_{\Sigma} \in(0, r(0))$ such that $\omega_{\Sigma}\left(r_{\Sigma}\right)=0$ and define $\operatorname{Dom}(R)=\{y \in$ $\left.\operatorname{Dom}\left(\omega_{\Sigma}\right):\left|\omega_{\Sigma}(y)\right| \leq\left|\omega_{\Sigma}(R)\right|\right\}$. If

$$
\begin{aligned}
\omega_{\Sigma}(y) \frac{d \omega_{\Sigma}}{d y}(y) & \geq 0 & & \text { for all } y \in \operatorname{Dom}(R), \\
\frac{d \omega_{\Sigma}}{d y}(y) & \neq 0 & & \text { for all } y \in \operatorname{Dom}(R),
\end{aligned}
$$

and $\omega_{0}$ satisfies the compatibility condition $\left.H\left(\omega_{0}\right)\right|_{y=R}=0$, then there exists a solution to the problem (2) for all times with $|\omega(y, t)|<\left|\omega_{\Sigma}(R)\right|$ for all $(y, t)$ and it converges as $t \rightarrow \infty$ to a minimal surface.

In some cases the long time existence can be strengthened to a convergence to constant functions result.

Theorem 1.3 (Convergence to a constant). Under the assumptions of Theorem 1.1:

(a) the solution of the problem (1) converges to a constant function as $t \rightarrow \infty$;

(b) if $z_{0}=0$ the solution of the problem (2) converges to the annulus $\left[r_{\Sigma}, R\right]$ as $t \rightarrow \infty$, where $r_{\Sigma}$ is such that $\omega_{\Sigma}\left(r_{\Sigma}\right)=0$. 
Our next result applies to the combined free Neumann boundary and fixed Dirichlet height problem. We state here the most general form of this result and later on give explicit sufficient conditions for initial graphs to satisfy the hypothesis of this theorem.

Theorem 1.4 (Curvature singularity on the boundary). Suppose $\Sigma$ is such that $\operatorname{Dom}\left(\omega_{\Sigma}\right)=\left[0, r_{\Sigma}\right]$ with $\omega_{\Sigma}\left(r_{\Sigma}\right)=0$ and satisfying in addition:

$$
\begin{aligned}
\omega_{\Sigma}(y) \frac{d \omega_{\Sigma}}{d y}(y) & \leq 0 & & \text { for all } y \in \operatorname{Dom}\left(\omega_{\Sigma}\right), \\
\frac{d \omega_{\Sigma}}{d y}(y) & \neq 0 & & \text { for all } y \in \operatorname{Dom}\left(\omega_{\Sigma}\right) \sim\{0\}, \\
\frac{d \omega_{\Sigma}}{d y}(0) & =0 . & &
\end{aligned}
$$

Let $\omega$ satisfy (2) on a domain $(r(t), R)$ such that $D(0)=(r(0), R), R \notin$ $\operatorname{Dom}\left(\omega_{\Sigma}\right)$ and $r(0) \leq r_{\Sigma}<R$. Suppose also that $\sup _{D(0)}\left|\omega_{0}\right|>\left|\omega_{\Sigma}(0)\right|$. If there exists a self-similar torus in the region of $\mathbb{R}^{n+1}$ defined as $\left\{\left(x_{1}, \ldots, x_{n+1}\right)\right.$ : $\left.\left|\omega_{\Sigma}(0)\right|<\left|x_{n+1}\right|<\left|\omega_{0}(y)\right|\right\}$ then the solution for the problem (2) exists for only a finite time $T<\infty$ and the graphs $\omega(\cdot, t)$ develop a curvature singularity at $y=0$ as $t \rightarrow T$. Also there exists a positive constant $C<\infty$ such that the singularity has the following rate of convergence:

$$
\|A\|_{\infty}^{2}(y) \leq \frac{C}{\left(\frac{d \omega_{\Sigma}}{d y}(0)\right)^{2}} \frac{1}{T-t},
$$

where we have denoted by $|A|$ the norm of the second fundamental form of the hypersurfaces evolving by mean curvature flow generated by the rotation of $\omega$ graphs.

We invite the reader to follow Section 5 on the proof of this theorem for more detailed conditions on initial graphs which permit the existence of a self similar torus in the above mentioned regions.

\section{Short time existence and boundary condition}

In this section we discuss the result of local existence and simplify the Neumann boundary condition.

The ninety degree contact angle condition on the fixed hypersurface $\Sigma$ which appears in the Neumann condition $\left\langle\nu_{\omega}, \nu_{\Sigma}\right\rangle=0$ can be written in a simpler way if we take into account that we are working with two graph functions. The outer normals to $\omega$ and $\omega_{\Sigma}$ are $\nu_{\omega}=\frac{1}{\sqrt{1+\left(\frac{d \omega}{d y}\right)^{2}}}\left(-\frac{d \omega}{d y}, 1\right)$ and $\nu_{\Sigma}=\frac{1}{\sqrt{1+\left(\frac{d \omega_{\Sigma}}{d y}\right)^{2}}}\left(-\frac{d \omega_{\Sigma}}{d y}, 1\right)$ respectively. This transforms our Neumann boundary condition to

$$
\frac{d \omega}{d y}(r(t))=-\frac{1}{\frac{d \omega_{\Sigma}}{d y}}(r(t)) \quad \text { for all } t \in[0, T) .
$$


The first step is to ensure that the two problems stated before at least exist for a short time. Since both (1) and (2) are quasilinear parabolic partial differential equations, short time existence has been heavily investigated in a large number of works, such as [12]. One might be tempted to also use previous results of mean curvature flow of graphs, such as [10]. The only problem that might arise is the fact that we are considering a time dependent domain which can cause the appearance of corners and other difficulties in the space time domain.

Since this forms part of the author's thesis we briefly state the result and only give an idea of the proof. The interested reader is invited to find the full detailed version in [14] for the case of general graphs.

Theorem 2.1 (Short time existence). For any $\alpha \in(0,1)$ there exists a positive time $\epsilon$ such that we have a solution $\omega$ for the problems (1) and (2) with $\omega \in$ $H_{2+\alpha}^{(-\delta)}(D(t) \times[0, \epsilon])$ for some $\delta \in(1,2)$.

Proof. The proof is based on the idea that we can always transform the domain of our problem from time dependent to time independent. This is done by choosing to use a vector field tangent to $\Sigma$ as the graph direction. By doing this, one ensures that we can apply results of short time existence such as those found in [12].

\section{Proof of the long time existence theorems}

The proof of Theorem 1.1 is based on the usual strategy of obtaining uniform height and gradient bounds. Since the underlying parabolic theory has been extensively treated throughout the literature we are here only concerned with providing the reader with a proof of how one might obtain the requisite height and gradient bounds. We start with the height bounds. The following result is valid for both case $(a)$ and case $(b)$ of Theorem 1.1 .

Lemma 3.1 (Height bound). If $\omega$ satisfies (1) or (2) in the domain $D(t)$ and the hypotheses of Theorem 1.1, then we have $\sup |\omega(y, t)| \leq \sup \left|\omega_{0}\right|$ for all $t$.

$$
D(t) \quad D(0)
$$

Proof. The maximum principle for time dependent domains as for example in [12] applied to the quasilinear parabolic evolution of $\omega$ gives us:

$\sup _{D(t)}|\omega(y, t)| \leq \max \left\{\sup _{D(0)}\left|\omega_{0}\right|, \sup _{s \in[0, t]} \sup _{y \in \partial D_{N}(s)}|\omega(y, s)|, \sup _{s \in[0, t]} \sup _{y \in \partial D_{D}}|\omega(y, s)|\right\}$,

for all $t \in[0, T]$. In case $(a)$, the term appearing from the Dirichlet boundary does not exist. Since we know our Dirichlet boundary values are constant in time and equal to $z_{0}$ (which is less than or equal to sup $\left|\omega_{0}\right|$ the last one of the $D(0)$

quantities in the right maximum from above can be ignored regardless. The only term we need to worry about is the one on the free Neumann boundary. Using (6), we shall compute the derivative in the direction normal to the 
Neumann boundary by taking the outer normal to the boundary in the two different cases of domain.

First, let us look at the problem (1) where the domain is defined as $D(t)=$ $(0, r(t))$. Here the choice of outer unit normal to the Neumann boundary is $\nu_{\partial D_{N}(t)}=\frac{y}{|y|}=1$ and then the directional derivative in the direction of the outer unit normal to the Neumann boundary is:

$$
\frac{d \omega}{d \nu_{D_{N}(t)}}=-\frac{1}{\frac{d \omega_{\Sigma}}{d y}} .
$$

If we find ourselves in the positive part of the $\Sigma$ surface then we see that the condition (3) says that $\frac{d \omega_{\Sigma}}{d y} \geq 0$, so we can put a sign on our directional derivative from above

$$
\frac{d \omega}{d \nu_{D_{N}(t)}} \leq 0
$$

If we assume that for some time $s \in[0, t]$ there exists a maximum of the graph function on the Neumann boundary then the Hopf Lemma tells us that the above computed directional derivative should be strictly positive, which contradicts (7). So we have no maxima on the Neumann boundary where $\omega \geq 0$. The same argument also applies when $\omega \leq 0$. This tells us that the absolute value of $\omega$ can not attain a maximum on the Neumann boundary. On the $y=0$ boundary point found on the axis of rotation, the same applies since the condition imposed on the directional derivative and the Hopf Lemma prevent both minima and maxima from appearing at this boundary point.

In case $(b)$ we have the domain $(r(t), R)$, so the unit outer normal to the Neumann boundary of this domain is of the opposite sign to the one in the first problem. Here the same argument applies using now condition (4).

Therefore we have demonstrated the required height bound in each case and so ends our proof.

We now turn our attention to gradient bounds.

Lemma 3.2 (Gradient bound). If $\omega$ satisfies (1) or (2) on the domain $D(t)$ and the hypotheses of Theorem 1.1, then there exists a global constant $C=$ $C\left(\omega_{0}, \Sigma\right)<\infty$ such that we have

$$
\sup _{D(t)}\left|\frac{d \omega}{d y}(y, t)\right| \leq C \quad \text { for all } t \in[0, T)
$$

Proof. Following [6] we consider the quantity $v=\left\langle\nu_{M_{t}}, e_{n+1}\right\rangle^{-1}$ which is equal up to tangential diffeomorphisms to $\sqrt{1+\left(\frac{d \omega}{d y}\right)^{2}}$. The function $v$ enjoys a parabolic evolution on the hypersurfaces $M_{t}$ generated by the graphs $\omega:$

$$
\left(\frac{d}{d t}-\Delta_{M_{t}}\right) v \leq 0
$$


and this allows us to apply the maximum principle. Since the problem deals with evolving hypersurfaces with boundary we have that the maximum of the gradient is controlled by the maximum between the initial values and the boundary values

$$
\sup _{D(t)} v \leq \max \left\{\sup _{D(0)} v, \sup _{s \in[0, t]} \sup _{\partial D_{N}(s)} v, \sup _{s \in[0, t]} \sup _{\partial D_{D}} v\right\},
$$

for all $t \in[0, T]$. The two boundary maximums can be bounded as follows. Following the work of Huisken [10] a barrier construction provides us with the required bound on $\partial D_{D}$ in a standard way.

On the Neumann boundary, the rotational symmetry of the solution (and the fact that this is preserved) prevents tilt behaviour. This occurs when the normal to the graph becomes parallel to the vector field of rotation for $\Sigma$. This behaviour is explained in much greater detail in [14].

Apart from this, we must argue why it is that our rotationally symmetric graph does not reach points on the Neumann boundary where the surface $\Sigma$ is horizontal. In such points the boundary gradient becomes infinite simply by the Neumann condition (6). To avoid such behaviour we combine Lemma 3.1 with the initial conditions from the long time existence theorem: either condition (3) for the purely Neumann problem (1) or condition (4) for the combined Dirichlet and Neumann problem (2). These conditions ensure that on the Neumann boundary, in the area enclosed by the maximum and minimum of the initial graph $\operatorname{Dom}(C)$, there is no point where $\Sigma$ becomes horizontal. Now since Lemma 3.1 implies that the height at later times remains bounded by the initial height, this continues to hold and the graph is bounded away from these potentially troublesome areas of $\Sigma$. This completes our proof.

Remark. In general, one can not prevent a curvature singularity from occurring on the free boundary without a condition such as (3) or (4). An example of such behaviour is given in Theorem 1.4.

Remark. Theorem 1.1 applies for example to radially symmetric graphs moving inside the catenoid neck (case (a)) and motion outside the unit sphere (case (b)).

Convergence to minimal surfaces follows from long time existence and the fact that we enjoy a uniform area bound. This has been detailed in [10]. A general version for immersions with free boundaries evolving by mean curvature flow can be also found in the author's thesis [14]. This finishes the proof of Theorem 1.1.

We now present the proof of the second theorem of long time existence.

Remark. Theorem 1.2 applies for example to the motion of radially symmetric graphs outside a catenoid neck with a fixed Dirichlet height on a circle of radius $R$.

Proof of Theorem 1.2. We are again concerned with obtaining uniform a priori height and gradient bounds. This time we are not able to prove that the height 
remains bounded by initial values as before. But still we are able to obtain gradient bounds and, using this, a height bound. One should keep in mind the picture of a solution evolving outside the neck of a catenoid with a fixed Dirichlet boundary at radius $R$. There it is intuitively obvious that a gradient bound implies a height bound: if the height of the solution grows without bound then it must 'cross itself', thus losing the graph property on the interior.

As before we make use of the function $v$ associated with our evolving graphs. The maximum principle implies

$$
\sup _{D(t)} v \leq \max \left\{\sup _{D(0)} v, \sup _{s \in[0, t]} \sup _{\partial D_{N}(s)} v, \sup _{s \in[0, t] \partial D_{D}} v\right\},
$$

for all times $t \in[0, T]$. The term on the Dirichlet boundary is bounded again by the usual construction of barriers [10]. The rotational symmetry and the Neumann boundary condition (6) imply also as before that the gradient does not become infinite on the Neumann boundary so long as the graph does not evolve towards a point where $\frac{d \omega_{\Sigma}}{d y}=0$.

In the argument earlier it was easy to exclude such behaviour by assuming that $\Sigma$ does not contain such points in the region between the maximum and minimum of the initial height and using the fact that the height remains bounded by initial values. Again here we prove that the solution only moves in a region where there are no points with $\frac{d \omega_{\Sigma}}{d y}=0$. Condition (5) implies that such a region is $\operatorname{Dom}(R)$. We know that our graph is initially defined on $D(0)=(r(0), R) \subset \operatorname{Dom}(R)$ with $r_{\Sigma}<r(0)<R$, and so our strategy is to show that the domain of definition of the moving graphs is contained within this domain for all times and thus that the gradient on the Neumann boundary remains bounded. From condition (5) one observes that $\operatorname{Dom}(R)=\left[r_{\Sigma}, R\right]$ where $\omega_{\Sigma}\left(r_{\Sigma}\right)=0$.

Let us pass temporarily to the more general setting of rotationally symmetric immersions, where we consider a unit speed curve $\gamma_{t}:[0, L] \rightarrow \mathbb{R}^{2}$ as the profile of revolution. Here $L$ depends also on time, but we suppress this for ease of exposition throughout the proof. We assume that there exists a time $\hat{t}$ such that the evolving hypersurface passes over the Dirichlet boundary, and shall bring this to a contradiction. This will prove that the domain of definition of $\omega$ is included in $\operatorname{Dom}(R)$.

In this planar setting consider the orthonormal frame which defines the coordinate system as $\left\{e_{1}, e_{2}\right\}$, where $e_{1}$ is the unit vector generating the $y$ axis and $e_{2}$ generates the vertical axis of rotation. Let us consider the set $\operatorname{Dom}(\gamma(\cdot, t)):=\left\{y: y=\left\langle\gamma(s, t), e_{1}\right\rangle\right.$ for some $\left.s \in[0, L]\right\}$. Our goal is to show that $\sup \operatorname{Dom}(\gamma(\cdot, t)) \leq R$. We first note that $\operatorname{Dom}(\gamma(\cdot, t))$ could not extend to the left beyond $r_{\Sigma}$. Our assumption is equivalent to assuming that $\sup \operatorname{Dom}(\gamma(\cdot, \hat{t}))>R$. This implies that the line $\{y=R\}$ intersects the image $\gamma([0, L], \hat{t})$ in at least two places, since we always have $\gamma(0, \hat{t})=(R, 0)$ by the Dirichlet boundary condition. That is, there exists $s_{1} \in[0, L]$ with $s_{1} \neq 0$ and $\left\langle\gamma\left(s_{1}, \hat{t}\right), e_{1}\right\rangle=\left\langle\gamma(0, \hat{t}), e_{1}\right\rangle=R$. It is important to keep in mind that on both boundaries the curve is not vertical, that is, $\left\langle\gamma^{\prime}(0, \hat{t}), e_{1}\right\rangle \neq 0$ and 
$\left\langle\gamma^{\prime}(L, \hat{t}), e_{1}\right\rangle \neq 0$. This means that if the graph representation were valid, $\omega(\cdot, \hat{t})$ would still have had bounded gradient on the Neumann and Dirichlet boundaries.

Define the distance from $\gamma$ to the vertical line at $R$ to be $d_{t}:[0, L] \rightarrow \mathbb{R}$, $d_{t}(s)=R-\left\langle\gamma(s, t), e_{1}\right\rangle$. Note that $d_{\hat{t}}(0)=d_{\hat{t}}\left(s_{1}\right)=0$. Since $\gamma$ is a regular curve, it has a well-defined tangent vector, and so $d_{t}$ is a $C^{1}$ function. Using this we obtain that there exists an $s^{*} \in\left[0, s_{1}\right]$ such that $d_{\hat{t}}^{\prime}\left(s^{*}\right)=0$, which from the definition of $d$ implies that $\left\langle\gamma^{\prime}\left(s^{*}, \hat{t}\right), e_{1}\right\rangle=0$. So the tangent vector at $s^{*}$ to the curve $\gamma$ does not have any components in the $e_{1}$ direction, which means it must be completely vertical. Clearly this implies that the graph representation $\omega$ of the solution is not valid at $t=\hat{t}$. So let us consider the time

$$
t^{*}=\inf \{t>0: \omega \text { is not a valid graph representation }\}
$$

Let us assume that during the time interval $\left[0, t^{*}\right]$ the solution leaves $\operatorname{Dom}(R)$, since if it did not, then we have nothing to prove. If the solution has extended its domain beyond $\operatorname{Dom}(R)$ then the argument above shows that there is an $s^{*}$ for which the tangent vector to $\gamma\left(\cdot, t^{*}\right)$ at $s^{*}$ is vertical. This implies that there exists a sequence of times $\left(t_{j}\right)$ with $t_{j} \rightarrow t^{*}$ and corresponding sequence of points $\left(y_{j}\right)$ with $y_{j} \rightarrow\left\langle\gamma\left(s^{*}, t^{*}\right), e_{1}\right\rangle$. Since $t^{*}$ is the first time that the graph 'turned on itself', the graph representation $\omega$ is in fact valid for all $j$ and in particular we may use this sequence of times and points with the maximum principle to obtain our desired contradiction: the sequence $v\left(y_{j}, t_{j}\right)$ grows without bound. Since $v$ is bounded at the Neumann and Dirichlet boundaries this implies that for $j$ sufficiently large, say $j>J$ there is an interior point where $v\left(y_{J}, t_{J}\right)$ is greater than its value on the boundaries, contradicting the maximum principle.

Hence the domain of definition of the evolving graphs is always included in $\operatorname{Dom}(R)$, that is $D(t) \subset \operatorname{Dom}(R)$, and this gives us gradient estimates for the evolving graphs.

This argument also gives a height bound. The maximum principle implies

$\sup _{D(t)}|\omega(y, t)| \leq \max \left\{\sup _{D(0)}\left|\omega_{0}\right|, \sup _{s \in[0, t]} \sup _{y \in \partial D_{N}(s)}|\omega(y, s)|, \sup _{s \in[0, t]} \sup _{y \in \partial D_{D}}|\omega(y, s)|\right\}$

for all times $t \in[0, T]$. The initial height and the Dirichlet boundary height are bounded by $C$. From the discussion above we already know that the graphs never evolve outside the height $\omega_{\Sigma}(R)$ on the Neumann boundary. This implies

$$
\sup _{s \in[0, T)} \sup _{y \in D(s)}|\omega(y, s)| \leq \max \left\{C, \omega_{\Sigma}(R)\right\}=\omega_{\Sigma}(R)<\infty
$$

The convergence to minimal surfaces follows from the long time existence and the fact that we enjoy a uniform area bound. 


\section{Proof of the convergence theorem}

We now turn to proving the convergence result. In order to aid in the understanding of the arguments to come let us keep the following examples in mind.

Remark (Examples). Theorem 1.3 is applicable, for example, to the motion of radially symmetric graphs inside the catenoid neck (case (a)) and to the motion of radially symmetric graphs outside the sphere (case (b)). The first converges to the flat disc inside the catenoid neck with zero height. For the second example the result of the theorem tells us that the radially symmetric graphs converge to the annulus around the sphere.

Proof of Theorem 1.3. The idea of the proof is to construct an auxiliary function which can be seen as the sum of the time scaled value of our moving graphs and their initial maximum value. The behaviour of the function gives us information about the time behaviour of the oscillations of the symmetric graphs. By oscillations we mean the difference of the minimum and maximum at each time. An appropriate height bound on this function tells us that it grows at most linearly in time and thus the oscillations of our mean curvature flow symmetric graphs will decrease linearly in time. First let us construct our auxiliary rotationally symmetric function and prove that it satisfies a height bound for all times. The same function is used to obtain both $(a)$ and $(b)$, although the argument by necessity must differ slightly at some points. Let $g: D(t) \times[0, \infty) \rightarrow \mathbb{R}$ be defined by

$$
g(y, t)=t \omega^{2}(y, t)+\frac{1}{2} y^{2} \sup _{D(0)}\left|\omega_{0}\right|^{2} .
$$

By its definition and the previous long time existence results Theorem 1.1 we have that this function exists for all times $t<\infty$ and from its height bound we obtain the desired convergence as time approaches infinity. Let us prove the height bound. At $t=0$ and on the Dirichlet boundary we have

$$
g(y, 0)=\frac{1}{2} y^{2} \sup _{D(0)}\left|\omega_{0}\right|^{2}, \quad \text { and } \quad g(R, t)=\frac{1}{2} R^{2} \sup _{D(0)}\left|\omega_{0}\right|^{2},
$$

since $\omega(R, t) \equiv 0$ by the condition $z_{0}=0$. Consider the following quasilinear parabolic operator

$$
L=\frac{\partial}{\partial t}-\frac{1}{\left(\frac{d \omega}{d y}\right)^{2}+1} \frac{d^{2}}{d y^{2}}-\frac{n-1}{y} \frac{d}{d y}
$$

By the gradient bound, Lemma 3.2, the coefficients of this operator remain bounded in the interior of our domains. Using Lemma 3.1 we can verify that 
$g$ is a supersolution for this operator:

$$
\begin{aligned}
(L g)(y, t)= & 2 t \omega(y, t)(L \omega)(y, t)-\frac{\sup _{D(0)}\left|\omega_{0}\right|^{2}}{\left(\frac{d \omega}{d y}\right)^{2}+1} \\
& -2 t\left(\frac{d \omega}{d y}\right)^{2} \frac{1}{\left(\frac{d \omega}{d y}\right)^{2}+1}-(n-1) \sup _{D(0)}\left|\omega_{0}\right|^{2}+\omega^{2}(y, t) \\
\leq & \omega^{2}(y, t)-\sup _{D(0)}\left|\omega_{0}\right|^{2} \leq 0 .
\end{aligned}
$$

Thus by the maximum principle

$$
\begin{aligned}
\sup _{D(t)} g(y, t) \leq \max \{ & \sup _{D(0)} \frac{1}{2} y^{2} \sup _{D(0)}\left|\omega_{0}\right|^{2}, \sup _{s \in[0, t]} \sup _{y \in \partial D_{N}(s)} g(y, s), \\
& \left.\frac{1}{2} R^{2} \sup _{D(0)}\left|\omega_{0}\right|^{2}\right\},
\end{aligned}
$$

for all times $t \in[0, T]$. To exclude boundary maxima we apply a similar argument as in Lemma 3.1, by calculating the sign of the derivative of $g$ in the direction normal to the Neumann boundary. Let us first compute the derivative:

$$
\frac{d g}{d y}=2 t \omega \frac{d \omega}{d y}+y \sup _{D(0)}\left|\omega_{0}\right|^{2}
$$

Now using (6)

$$
\begin{aligned}
\frac{d g}{d \nu_{D_{N}}(t)} & =2 t \omega(r(t), t) \frac{d \omega}{d y}(r(t), t) \nu_{D_{N}(t)}+r(t) \sup _{D(0)}\left|\omega_{0}\right|^{2} \nu_{D_{N}(t)} \\
& =-2 t \omega_{\Sigma} \frac{1}{\frac{d \omega_{\Sigma}}{d y}} \nu_{D_{N}(t)}+r(t) \sup _{D(0)}\left|\omega_{0}\right|^{2} \nu_{D_{N}(t)} \\
& =-2 t \omega_{\Sigma}^{2} \frac{1}{\omega_{\Sigma} \frac{d \omega_{\Sigma}}{d y}} \nu_{D_{N}(t)}+r(t) \sup _{D(0)}\left|\omega_{0}\right|^{2} \nu_{D_{N}(t)} .
\end{aligned}
$$

Our argument must now differ for each of the cases $(a)$ and $(b)$. We begin with (a).

In the case of the Neumann problem (1), the domain is given by $D(t)=$ $(0, r(t))$ with boundary $\partial D_{N}(t)=r(t)$. The 0 point on the rotation axis is here regarded as a boundary point also. For a radially symmetric graph we have $\left.\frac{d \omega}{d y}\right|_{y=0}=0$ by smoothness and symmetry as explained before. The normal to the Neumann boundary $\partial D_{N}(t)$ is $\nu_{D_{N}(t)}=\frac{y}{|y|}=1$.

In this case we can not exclude the appearance of a maximum for $g$ on the Neumann boundary by contradicting condition (3) with the Hopf Lemma. Instead we proceed in a manner somewhat analagous to the proof of Theorem 1.2 above. 
First we shall dismiss the appearance of maxima at the point $y=0$ using a Hopf Lemma argument. From the boundary condition at the rotation axis we have

$$
\left.\frac{d g}{d y}\right|_{y=0}=\left.2 t \omega \frac{d \omega}{d y}\right|_{y=0}+\left.y \sup _{D(0)}\left|\omega_{0}\right|^{2}\right|_{y=0}=0 .
$$

Then the height of the $g$ function satisfies

$$
\sup _{D(t)} g(y, t) \leq \max \left\{\sup _{D(0)} \frac{1}{2} y^{2} \sup _{D(0)}\left|\omega_{0}\right|^{2}, \sup _{s \in[0, t]} \sup _{y \in \partial D_{N}(s)} g(y, s)\right\}
$$

for all times $t \in[0, T]$. Recall that we do not have a Dirichlet boundary term in the case of the problem (1). From Lemma 3.1 we obtain that the height of the function $g$ is bounded for all times $t<\infty$.

Replacing the definitions we can further compute

$$
\begin{aligned}
t \omega^{2}(y, t)+\frac{1}{2} y^{2} \sup _{D(0)}\left|\omega_{0}\right|^{2} \leq \max & \left\{\sup _{D(0)} \frac{1}{2} y^{2} \sup _{D(0)}\left|\omega_{0}\right|^{2},\right. \\
\left.\sup _{s \in[0, t]} \sup _{y \in \partial D_{N}(s)}\left\{s \omega^{2}(y, s)+\frac{1}{2} y^{2} \sup _{D(0)}\left|\omega_{0}\right|^{2}\right\}\right\} & \left\{\frac{1}{2} r(0)^{2} \sup _{D(0)}\left|\omega_{0}\right|^{2},\right. \\
t \omega^{2}(y, t)+\frac{1}{2} y^{2} \sup _{D(0)}\left|\omega_{0}\right|^{2} \leq \max & \left\{\sup _{s \in[0, t]} s \omega^{2}(r(s), s)+\frac{1}{2} r(s)^{2} \sup _{D(0)}\left|\omega_{0}\right|^{2}\right\} .
\end{aligned}
$$

Thus

$$
t \omega^{2}(y, t) \leq \tilde{C}+\sup _{s \in[0, t]} s \omega^{2}(r(s), s)
$$

for some positive constant $\tilde{C}<\infty$. Here we have used the fact that $r(t)$ and $y \in[0, r(t)]$ are bounded by positive constants and also the positivity of the terms found in the maximum. The above bound on $r(t)$ comes from the height bound result of Lemma 3.1 which gives us that $r(t) \in \operatorname{Dom}(C)$ where $\operatorname{Dom}(C)$ was taken to be a bounded domain. Therefore

$$
\omega^{2}(y, t) \leq \frac{C}{t}+\frac{1}{t} \sup _{s \in[0, t]} s \omega^{2}(r(s), s) .
$$

From Theorem 1.1 we have that there exists a smooth solution $\omega_{\infty}$ for the problem (1) for all times. This solution might not be unique as previous results in mean curvature flow with boundaries suggest [10]. Keeping this in mind when we denote by $\omega_{\infty}$ and $r(\infty)$ the limiting solution and the Neuman boundary point for this limiting solution respectively we think of a subsequence of times $t_{k} \rightarrow \infty$ such that $\omega\left(\cdot, t_{k}\right) \rightarrow \omega_{\infty}$. For the ease of exposition we abuse notation and consider that $r(t) \rightarrow r(\infty)$ as $t \rightarrow \infty$.

Depending on the value that the limiting solution has on the Neumann boundary we distinguish the following cases. If we $\omega_{\infty}(r(\infty)) \neq 0$ this implies 
that the solution was not vanishing on the boundary for some time before, that is being either strictly negative or strictly positive. So there exists $t^{*} \geq 0$ such that $\omega(\cdot, t) \neq 0$ for all $t \geq t^{*}$. Thus, since for all times the evolving graphs have bounded height, see Lemma 3.1, there exists a time $T^{*} \geq t^{*}$ such that $\sup _{s \in[0, t]} s \omega^{2}(r(s), s)=t \omega^{2}(r(t), t)$ for all $t \geq T^{*}$. In (13) we have

$$
\omega^{2}(y, t) \leq \frac{C}{t}+\omega^{2}(r(t), t)
$$

Take $t \rightarrow \infty$ to obtain

$$
\omega_{\infty}^{2}(y) \leq \omega_{\infty}^{2}(r(\infty))
$$

This give us a maximum of the height of the solution on the Neumann boundary at time $T=\infty$. The height bound Lemma 3.1 tell us that we can not have a height maximum on the Neumann boundary unless the solution is a constant function.

The second case treats the situation when $\omega_{\infty}(r(\infty))=0$. The above argument does not apply anymore since the supremum of the Neumann boundary values could have been obtained at a previous time. Our evolving graphs are defined on bounded domains, that is $\operatorname{Dom}(C)$ is taken to be bounded in Theorem 1.1 and thus generating rotationally symmetric surfaces evolving by mean curvature flow with Neumann boundary conditions. Following an idea of Huisken [10] one can show that, due to the long time existence of the solutions we have that the limiting surface or surfaces are minimal (as obtained in the long time existence theorems). That is mean curvature is vanishing on every point of the surface obtained at final time $\left.H\right|_{t=\infty}=: H_{\infty} \equiv 0$. Once again we mention that if the solution is not unique we restrict our work to the time subsequences in question. Let us denote by $u(x)=\omega_{\infty}(y)$ the general graph over $D_{\infty}=\left\{x \in \mathbb{R}^{n}: y=|x| \in \operatorname{Dom}\left(\omega_{\infty}\right)\right\}$ generated by $\omega_{\infty}$. Its mean curvature can be easily define to be $H=\operatorname{div}\left(\frac{D u}{\sqrt{1+|D u|^{2}}}\right)$. We can then compute

$$
\begin{aligned}
0 & =\int_{D_{\infty}} H u d x=\int_{D_{\infty}} d i v\left(\frac{D u}{\sqrt{1+|D u|^{2}}}\right) u d x \\
& =-\int_{D_{\infty}} \frac{|D u|^{2}}{\sqrt{1+|D u|^{2}}} d x+\int_{\partial D_{\infty}} D u \cdot \nu_{\partial D_{\infty}} u d S x \\
& =-\int_{D_{\infty}} \frac{|D u|^{2}}{\sqrt{1+|D u|^{2}}} d x
\end{aligned}
$$

where we have used $\omega_{\infty}(r(\infty))=0$ for the Neumann boundary (i.e. $u(x)=0$ for all $\left.x \in \partial D_{\infty}\right)$ and also the smoothness of the solution at the rotation axis, $\frac{d \omega_{\infty}}{d y}(0)=0$ to make the boundary term vanish. This implies that $D u \equiv 0$ and thus $\frac{d \omega_{\infty}}{d y} \equiv 0$ and proves that the solution has to be a constant function. The vanishing value on the Neumann boundary also tells us that in this case we not only have a constant function but the function is completely vanishing. 
We now turn our attention to case $(b)$. For the problem (2) we have two boundary conditions, a Neumann and a Dirichlet. The domain is given by $D(t)=(r(t), R)$ with the boundaries being $\partial D_{D}=R$ and $\partial D_{N}(t)=r(t)$. Here the situation is easier than in the previous case since on the Neumann boundary we can prove directly that we do not have any maximum of the function $g$. This comes from (11) together with the fact that the outer normal to the Neumann boundary is $\nu_{\partial D_{N}(t)}=-\frac{y}{|y|}=-1$ :

$$
\frac{d g}{d \nu_{D_{N}}(t)}=2 t \omega_{\Sigma}^{2} \frac{1}{\omega_{\Sigma} \frac{d \omega_{\Sigma}}{d y}}-r(t) \sup _{D(0)}\left|\omega_{0}\right|^{2} \leq 0,
$$

where the last inequality is implied by condition (4).

The Hopf Lemma tells us then that there does not exist any maximuma of $g$ on the Neumann boundary. The previous application of the maximum principle in (9) gives us that here the height of $g$ is bounded by the maximum between initial values and values on the Dirichlet boundary. This implies

$$
\begin{aligned}
t \omega^{2}(y, t)+\frac{1}{2} y^{2} \sup _{D(0)}\left|\omega_{0}\right|^{2} & \leq \max \left\{\frac{1}{2} R^{2} \sup _{D(0)}\left|\omega_{0}\right|^{2}, \sup _{(r(0), R)} \frac{1}{2} y^{2} \sup _{D(0)}\left|\omega_{0}\right|^{2}\right\} \\
& \leq \frac{1}{2} R^{2} \sup _{D(0)}\left|\omega_{0}\right|^{2}
\end{aligned}
$$

which leads to

$$
\omega^{2}(y, t) \leq \frac{1}{2 t}\left(R^{2}-y^{2}\right) \sup _{D(0)}\left|\omega_{0}\right|^{2}, \text { for every } t<\infty .
$$

Taking $t \rightarrow \infty$ in the last line gives us that $\omega^{2}(y, t) \leq 0$ as $t \rightarrow \infty$. This implies $\omega$ converges to the zero function defined over an annulus. This is of course the annulus itself and ends the second part of our proof.

Remark ( $C^{\infty}$ convergence). The convergence in the above theorem is only in the $C^{0}$ topology, since we have only explicitly shown that the height converges to a constant as $t \rightarrow \infty$. To obtain convergence in the $C^{\infty}$ topology, where all derivatives must also converge, one must apply interior estimates (such as can be found in $[5,7])$ after one has already established long time existence.

\section{Curvature singularity on the boundary}

The results of this section only apply to problem (2). We use a self-similar solution of mean curvature flow to show that for some specific class of initial data the graphs evolve towards the rotation axis $y=0$ where the surface $\Sigma$ is horizontal, developing a curvature singularity by being pinched. We give the proof of Theorem 1.4 and in the next subsection we present sufficient conditions for the initial graph to ultimately display such behaviour at the final time. 


\subsection{The singularity theorem}

In Theorem 1.1 we gave sufficient conditions for which the height of the graphs for both of our problems (1) and (2) remains bounded by the initial values. The initial conditions also include a relation which states that between the maximum and minimum of the initial height there is no point on which the surface $\Sigma$ becomes horizontal. These two conditions are enough to prevent the graphs $\omega$ from developing an infinite gradient on the Neumann boundary.

When the surface $\Sigma$ has a point in which it is horizontal in the above mentioned region, that is there exists a point between the maximum and minimum of the height of the initial graph such that $\frac{d \omega_{\Sigma}}{d y}=0\left(\right.$ or $\left.\left|\left\langle\nu_{\Sigma}, e_{n+1}\right\rangle\right|=1\right)$, then there is essentially no obstruction to the evolving graphs moving towards those points. When one of these points lie upon the axis of rotation for $\Sigma$, a curvature singularity can also develop. This is the case which interests us in this section.

In the following, as required by the hypothesis of the theorem we consider rotationally symmetric surfaces $\Sigma$ which are diffeomorphic to spheres with rotation axis $y=0$.

Proof of Theorem 1.4. We apply the comparison principle to obtain that the moving graphs and the enclosed self-similar torus never touch.

Note that our previous results imply that the solution $\omega$ continues to exist until the appearance of the first gradient singularity on the boundary. Due to the rotational symmetry and the maximum principle, this is only possible on the Neumann boundary. This ensures that the solution exists at least for as long as the torus beneath (or above) it.

Denote by $X_{t}$ an Angenent torus, that is a self-similar shrinking solution of mean curvature flow. Let $M_{t} \subset \mathbb{R}^{n+1}$ be the hypersurface evolving by mean curvature flow generated by radially symmetric graphs, that is $M_{t}=$ $(x, \omega(|x|, t))$. From the hypothesis of the theorem we have that at initial time the torus and the evolving hypersurfaces are disjoint $M_{0} \cup X_{0}=\emptyset$ and also we have that the boundary of $M_{0}$ is outside the set $\mathcal{S}=\left\{\left(x_{1}, \ldots, x_{n+1}\right)\right.$ : $\left.\left|\omega_{\Sigma}(0)\right|<\left|x_{n+1}\right|<\left|\omega_{0}(y)\right|\right\}$ which contains the initial torus.

The boundary point value is $|\omega(r(t), t)|<\left|\omega_{\Sigma}(0)\right|$ for any $r(t) \neq 0$ and this shows us that the boundary of $M_{t}$ is outside the $\mathcal{S}$ set for all times. The torus evolves self-similarly so if it is initially contained in the set $\mathcal{S}$ it will remain in the set for all times of existence. Thus the torus will never reach the boundary of the evolving graphs, implying that a compact version of the comparison principle is enough for our argument. Using the comparison principle developed by Huisken [9] for mean curvature flow solutions we prove that our moving graphs and the evolving tori remain disjoint for all time, $M_{t} \cup X_{t}=\emptyset$ for all times $t \geq 0$. By our choice of a self-similar shrinking torus we have that it exists for a finite quantum of time $T$ until it becomes a point, forcing the solution to pinch at the point $y=0$ as $t \rightarrow T$.

To prove the rate of convergence we denote by $F$ the one-parameter family of smooth embeddings for the hypersurfaces $M_{t}$. That is $F(x, t)=(x, \omega(|x|, t))$. 
The principle curvatures can be computed as

$$
k_{i}=-\frac{1}{\sqrt{1+\left(\frac{d \omega}{d y}\right)^{2}}}\left(\frac{x_{i}^{2}}{|x|^{2}} \frac{d^{2} \omega}{d y^{2}}(y)+\frac{d \omega}{d y}(y)\left(\frac{1}{|x|^{2}}-\frac{x_{i}^{2}}{|x|^{3}}\right)\right),
$$

for all $i=1 \ldots n$ and where we recall the notation $y=|x|$. We can compute the second fundamental form squared as the sum of the square of the principle curvatures as follow

$$
|A|^{2}=\sum_{i=1}^{n} k_{i}^{2} \leq\left(\frac{d^{2} \omega}{d y^{2}}\right)^{2}(y)+\frac{1}{|x|^{2}}\left(\frac{d \omega}{d y}\right)^{2}(y) .
$$

Due to the quasilinear uniformly parabolic nature of our evolution equation in the case of uniform gradient bounds, we can also bound the second derivatives in terms of the gradient and the height. To show that one can assume the contrary on any finite time interval. The uniform gradient bound and parabolic theory will imply $C^{2+\alpha}$ bounds, for some $\alpha \in(0,1)$ and contradict the assumption. From Lemma 3.1 we have that the height is bounded by initial values and thus there exists a positive constant $C_{1}<\infty$ such that

$$
\|A\|_{\infty}^{2} \leq \sup _{y \in D(t)} \frac{C_{1}}{y^{2}}\left(\frac{d \omega}{d y}\right)^{2}(y)
$$

First we use the comparison principle to show that the radius of rotation shrinks no faster than the radius of a shrinking sphere. This time we place a sphere centered on the rotation axis above the maximum height of $\Sigma$, or below the minimum height if we find ourselves on the negative side of the graphs $\omega_{\Sigma}$. Taking the initial sphere to be disjoint from the evolving graphs and just like the torus, above the boundary point, one obtains that they remain disjoint for all times of existence. Therefore the rotation radius is shrinking at most as fast as the radius of the sphere. Now since a sphere evolving by mean curvature flow develops a Type I curvature singularity when it finally contracts at the center point on the rotation axis $y=0$ (in fact we could place any strictly convex surface there, as one can infer from [9], or even any strictly convex surface meeting $\Sigma$ perpendicularly, as one can infer from [13]) and since we have that the second fundamental form of the sphere is inverse proportional to the radius squared of sphere we have that there exists a positive constant $C_{2}<\infty$ such that

$$
\frac{1}{y^{2}} \leq \frac{C_{2}}{T-t}
$$

Also using the Neumann boundary condition (6) we obtain that at the boundary singularity point the second fundamental form satisfies

$$
\|A\|_{\infty}^{2} \leq \frac{C}{\left(\frac{d \omega_{\Sigma}}{d y}(0)\right)^{2}} \frac{1}{T-t} .
$$

for some positive constant $C=C\left(C_{1}, C_{2}\right)<\infty$. 
Remark (Example of rate of convergence). If our hypersurface of contact $\Sigma$ is the unit sphere centred at the origin we can approximate the rate of convergence for the singularity as

$$
\left(\frac{d \omega_{\Sigma}}{d y}(y)\right)^{2}=\frac{y^{2}}{1-y^{2}} \geq y^{2} \geq \frac{C}{T-t}
$$

In the last inequality we have, just like in the proof above, used the comparison principle with a shrinking sphere evolving by mean curvature flow. This gives us the following rate of convergence.

$$
\|A\|_{\infty}^{2} \leq \frac{C}{(T-t)^{2}}
$$

for some positive constant $C<\infty$.

Remark (Type I singularity). If in the hypothesis of the theorem we replace the condition $\frac{d \omega_{\Sigma}}{d y}(0)=0$ with $\left|\lim _{y \rightarrow 0} \frac{d \omega_{\Sigma}}{d y}(y)\right| \geq C$ for some positive constant $0<C<\infty$ then the evolving $\omega$ graphs develop a Type I singularity on the rotation axis. The condition for the $\Sigma$ graphs implies that the $\Sigma$ hypersurface is not smooth. As an example we can consider $\Sigma$ to be a cone.

\subsection{Sufficient conditions on initial graphs}

In the previous proof we have made use of the existence of a self-similar solution of mean curvature flow. In the following we want to use the previous work done by Angenent to provide the reader with exact sufficient conditions imposed on our rotationally $n$-dimensional graphs such that Theorem 1.4 can be applied.

The following result due to Angenent proves the existence of such a selfsimilar torus. [2].

Theorem 5.1 (Angenent [2], 1989). For $n \geq 2$ there exist embeddings $X_{n}$ : $S^{1} \times S^{n-1} \rightarrow \mathbb{R}^{n+1}$ for which $X_{n}(p, t)=\sqrt{2(1-t)} \cdot X_{n}(p)$ is a solution of the flow by mean curvature equation.

This theorem states the existence of a self-similar torus. The solution for the compact mean curvature flow given by the embedding in the theorem above states that the torus shrinks to the origin by dilatations, and it will become singular at time $T=1$.

There are three quantities which characterise a torus, and Angenent finds conditions on these to ensure the torus is self similar. We consider the torus as a hypersurface of revolution. The width of the torus is the maximal distance from the rotation axis taken pointwise, and the radius of the hole is the minimum of all distances of points from the curve to the axis of rotation. The third quantity is the 'fatness' of the torus or the maximum height that the plane curve takes as a graph. Let us denote the three quantities by $r_{1}$ for the radius of the hole, $r_{2}$ for the width of the torus, and $h$ for the maximum height. In 
case we are looking at a perfect torus, obtained by the rotation of a circle, the three quantities are: $r_{1}$ is the difference between the radius of the rotation and the radius of the rotated circle, $r_{2}$ is the sum of $r_{1}$ and the diameter of the rotated circle and $h$ is just the radius of the circle which is being rotated. Following the work of Angenent [2] one finds, quite surprisingly, that the selfsimilar torus is not a 'perfect torus', obtained by the rotation of a circle. It is in fact somewhat egg-shaped, so working directly with the self-similar torus is not easy. This is why we make use of a little trick.

Since we are working with a general graph we want to give the most general condition on the three quantities for which the existence of a self-similar torus is assured. The most direct method is to fit a big "box" in the region where we wish to place the curve generating the self-similar torus, which then is contained in the "box". From discussions found in [2] we obtain relations between the three quantities, for which the torus is a self-similar shrinking solution.

Lemma 5.2 (Angenent [2], 1989). There exists a smallest $r_{2}^{*} \geq \sqrt{2 n}$ for which we have a self-similar torus with $r_{1}^{*} \geq \frac{1}{r_{2}^{*}}$ and $h \leq \frac{C}{r_{2}^{*}}$, where $C$ does not depend on $r_{2}^{*}$.

For $n=2$ one can also estimate numerically $[2,4]$ the approximate values of these three quantities for the self-similar torus.

Remark (Angenent [2], 1989, Chopp [4], 1994, Approximate values for the self-similar torus for $n=2)$. For the above existence result in the case $n=2$ we have the following approximate values

$$
r_{2}^{*}=3.4, r_{1}^{*}=0.45, h^{*}=0.87 \text { and } C<3,
$$

with extinction time $T=1$.

Of course one may always find a "smaller" torus which also shrinks selfsimilarly. Thus we must be very careful in the scaling behaviour of these three quantities. In the following we look at the conditions that a scaled version of the self-similar torus must satisfy. Assuming we start with another value for the width of the torus, which we denote as before with $r_{2}$, we need to introduce a scaling factor:

$$
\lambda=\frac{r_{2}}{r_{2}^{*}} \leq \frac{r_{2}}{\sqrt{2 n}}
$$

The conditions imposed on the three quantities are scaled appropriately as:

(a) $r_{1} \geq \lambda^{2} \frac{1}{r_{2}}$,

(b) $h \leq \lambda^{2} \frac{C}{r_{2}}$.

As announced in the lemma above, note that the constant $C$ does not depend on the scaling.

Next we wish to give sufficient conditions for the self-similar torus of Theorem 1.4 to exist. We work assuming that the initial graph is positive. The negative or mixed cases are treated similarly. Let us set

$$
\mathcal{Q}=\left\{z \in \mathbb{R}: \exists y \in[r(0), R] \text { such that } z=\omega_{0}(y) \text { and } z>\omega_{\Sigma}(0)\right\}
$$


to be the set of all initial values above the fixed height line $z=\omega_{\Sigma}(0)$ from Theorem 1.4. Let

$$
M=\sup _{z \in \mathcal{Q}} \omega_{0}^{-1}(z)
$$

be the farthest point away from the rotation axis $y=0$ for which the initial graph is above the maximal value of the $\Sigma$ graphs. Then we have the following proposition.

Proposition 5.3 (Conditions on the initial graph). Suppose $\omega_{0}$ is an initial graph for the problem (2). If there exists $0<R_{2} \leq M$ and there exist $z_{1}, z_{2} \in \mathcal{Q}$ with $z_{1} \leq z_{2}$ and $z_{2}-z_{1} \geq C \frac{2 R_{2}}{r_{2}^{* 2}}$ such that $\max \omega_{0}^{-1}(z)-\min \omega_{0}^{-1}(z) \geq$ $R_{2}-\frac{R_{2}}{r_{2}^{* 2}}$ for all $z \in\left[z_{1}, z_{2}\right]$, then there exists the self-similar torus required for Theorem 1.4.

Proof. The two conditions found in the hypothesis are sufficient to enable us to construct a "box" high enough and wide enough such that we are able to fit the curve which generates the self-similar torus in the region bounded by the constant height $z=\omega_{\Sigma}(0)$ and the initial graph $\omega_{0}$. The box permits our curve to satisfy the above scaled conditions (a) and (b).

\section{Applications}

We wish to mention here two examples for problems (1) and (2). The first is the motion by mean curvature flow of radially symmetric graphs inside the catenoid neck, and the second the motion by mean curvature flow of radially symmetric graphs outside the unit sphere combined with a fixed zero Dirichlet boundary height at a fixed radius outside the sphere. In the first problem our results imply that the mean curvature flow solution exists for all times and converges to the flat disc inside the catenoid neck. For the second we have shown that the problem (2) has a long time solution if we start with an initial graph below the height of the sphere, and converges as $t \rightarrow \infty$ to the annulus around the sphere. In the case where we do not have such an initial bound on the height and instead satisfy the conditions of Theorem 1.4, the graphs move towards the North Pole of the sphere (or the South Pole in case we find ourselves with a negative graph). The graph develops a curvature singularity at either of these poles in finite time.

A third example which satisfies the hypothesis of Theorem 1.2 is the motion by mean curvature of radially symmetric graphs outside the catenoid neck with a ninety degree angle condition on the catenoid and a Dirichlet height on some circle of fixed radius outside the catenoid neck. Our theorem shows that the graphs exist for all time and converge to a minimal surface satisfying the same boundary conditions.

Acknowledgements The results contained in this paper are part of the author's Ph.D. thesis under the supervision of Prof. Klaus Ecker at the Free University in Berlin, in the group of Geometric Analysis as a Berlin Mathematical School student. The author would 
like to thank them for financial support and help during the completion of her thesis. This paper was written and completed during an extended visit to the University of Wollongong, Australia, as an invite of Prof. Graham Williams and Dr. James McCoy. The author is greatly indebted to them for their hospitality and advice during her stay, and would like to thank them for many enlightening discussions on this work. The author would also like to thank the anonymous referee whose careful reading and comments have led to an improvement of this paper.

\section{References}

1. Altschuler, S., Wu, L.: Translating surfaces of the non-parametric mean curvature flow with prescribed contact angle. Calc. Var. Partial Differential Equations 2, 101-111 (1994)

2. Angenent, S.: Shrinking doughnuts. Proc. of the Conf. on Elliptic and Parabolic Equations held at Gregynoy, Wales (1989)

3. Buckland, J.: Mean curvature flow with free boundary on smooth hypersurfaces. J. Reine Angew. Math. 586, 71-90 (2005)

4. Chopp, D.: Computation of self-similar solutions for mean curvature flow. Experiment. Math. 3(1), 1-15 (1994)

5. Colding, T., Minicozzi, W.: Sharp estimates for mean curvature flow of graphs. J. Reine Angew. Math. 574, 187-195 (2004)

6. Ecker, K., Huisken, G.: Mean curvature evolution of entire graphs. Ann. of Math. 130(2), 453-471 (1989)

7. Ecker, K., Huisken, G.: Interior estimates for hypersurfaces moving by mean curvature. Invent. Math. 105(1), 547-569 (1991)

8. Guan, B.: Mean curvature motion of nonparametric hypersurfaces with contact angle condition. Elliptic and parabolic methods in geometry p. 47 (1996)

9. Huisken, G.: Flow by mean curvature of convex surfaces into spheres. J. Differential Geom. 20(1), 237-266 (1984)

10. Huisken, G.: Non-parametric mean curvature evolution with boundary conditions. J. Differential Equations 77, 369-378 (1989)

11. Koeller, A.: On the Singularity Sets of Minimal Surfaces and a Mean Curvature Flow. Ph.D. thesis, Freie Universität Berlin (2007)

12. Lieberman, G.: Second order parabolic differential equations. World Scientific Pub. Co. Inc. (1996)

13. Stahl, A.: Über den mittleren Krümmungsfluss mit Neumannrandwerten auf glatten Hyperflächen. Ph.D. thesis, Fachbereich Mathematik, Eberhard-Karls-Universität, Tüebingen, Germany (1994)

14. Vulcanov, V.: Mean curvature flow of graphs with free boundaries. Ph.D. thesis, Freie Universität, Fachbereich Mathematik und Informatik, Berlin, Germany (2010) 